\title{
CRITICAL PERIODS FOR EFFECTS OF MONOCULAR DEPRIVATION: DIFFERENCES BETWEEN STRIATE AND EXTRASTRIATE CORTEX ${ }^{1}$
}

\author{
KIM R. JONES, ${ }^{2}$ PETER D. SPEAR, ${ }^{3}$ AND LILLIAN TONG \\ Department of Psychology, University of Wisconsin, Madison, Wisconsin 53706 \\ Received January 23, 1984; Revised March 30, 1984; Accepted April 16, 1984
}

\begin{abstract}
The critical period of susceptibility to effects of monocular deprivation was compared in striate cortex and the lateral suprasylvian (LS) visual area of cortex. Twenty-three cats received monocular lid suture for a period of 4 weeks beginning at $4,12,18,26$, or 35 weeks of age or as adults. Immediately following the deprivation, single cell recordings were carried out in both cortical areas of each cat. Recordings also were made from five normally reared control cats. For both striate and LS cortex, early monocular deprivation had marked effects on neuronal ocular dominance, including an increased percentage of cells dominated by the nondeprived eye, a decreased percentage of cells dominated by the deprived eye, and a decreased percentage of binocularly driven cells. In both cortical areas, these effects were maximal in animals deprived at 4 weeks of age. Both areas then showed similar monotonic declines in effects of the deprivation following onsets from 4 to 18 weeks of age. However, in older animals there were clear differences in the effects of monocular deprivation on LS and striate cortex. In LS cortex, the monotonic decline in effects continued until 26 weeks of age, and no significant abnormalities were present in animals deprived at 26 weeks of age or older. In striate cortex, however, the effects of monocular deprivation remained relatively constant following onsets from 18 to 35 weeks of age, and significant abnormalities in all measures of ocular dominance were present when deprivation was begun as late as 35 weeks of age. Within-animal comparisons indicated that the greater effects of monocular deprivation on striate cortex than on LS cortex were present in every cat deprived at 26 or 35 weeks of age. Neither cortical area showed significant abnormalities following monocular deprivation in adult cats. These results indicate that the critical period for effects of the same regime of monocular deprivation is over sooner in LS cortex (between 18 and 26 weeks of age) than in striate cortex (after 35 weeks of age). This observation has important implications for an understanding of the sites and mechanisms of effects of visual deprivation and the mechanisms that control critical periods of development.
\end{abstract}

One of the most important observations to arise from the voluminous research on effects of rearing with visual deprivation has been the demonstration of a critical period of development during which physiological properties of striate cortex (area 17) neurons can be modified by the environment (see Mitchell, 1981; Movshon and Van Sluyters, 1981, for reviews). For example, rearing kittens with monocular pattern deprivation produces a marked loss in the ability of the deprived eye to drive striate cortex neurons only if the deprivation occurs during a relatively limited period of early development (Hubel and Wiesel, 1970). A loss of response to the deprived eye

\footnotetext{
1 This work was supported by United States Public Health Service Postdoctoral Fellowships EY05449 (K. R. J.) and EY05256 (L. T.), Research Grants EY01916 and EY02545, and Research Career Development Award EY00089 (P. D. S.). We thank Ms. Kathy Vielhuber and Ms. Stephanie Miller for their excellent technical assistance.

${ }^{2}$ Present address: University of North Carolina School of Medicine, Chapel Hill, NC 27514.

${ }^{3}$ To whom reprint requests should be addressed, at Department of Psychology, University of Wisconsin, 1202 West Johnson Street, Madison, WI 53706.
}

following monocular deprivation also has been found in an extrastriate region of cortex, the lateral suprasylvian (LS) visual area (Spear and Tong, 1980). However, it is not known whether the critical period in this, or any other, cortical area is similar to that in striate cortex. This is an important question for understanding the neural mechanisms, and for interpreting the behavioral consequences, of early visual deprivation.

We were led to suspect that the critical period of plasticity might be different in LS cortex and striate cortex because of recent results concerning the critical period for functional compensation in LS cortex after neonatal visual cortex lesions. Tong et al. (1984) found that the ability of LS cortex neurons to develop normal receptive field and ocular dominance properties following early removal of areas 17 and 18 persists until sometime between the 18 th and the 26 th week of life. In contrast, studies that have investigated the effects of monocular deprivation or its reversal in striate cortex indicate that the modifiability of neurons in this area ends sometime between the 12th and the 14th week of life (Wiesel and Hubel, 1965; Hubel and Wiesel, 1970; Blakemore and Van Sluyters, 1974). This difference raised the possibility that LS and striate cortex might show general differences in their critical periods of plasticity. 
To compare these two areas as directly as possible, we subjected kittens of various ages to 4 weeks of monocular deprivation and then measured the ability of each eye to drive neurons in both LS and striate cortex of each cat. We also recorded from both areas in adult cats that had undergone an identical 4 weeks of monocular deprivation and in normal control cats.

\section{Materials and Methods}

Subjects. Sixteen kittens and seven adult cats were monocularly deprived by suturing closed the lids of one eye under halothane anesthesia. The procedure used is described elsewhere (Spear et al., 1980b; Spear and Tong, 1980). The kittens were divided into groups of three deprived at $4,12,18$, or 26 weeks of age, and a group of four, deprived at 35 weeks. Littermates were assigned to different age groups, and within each age group the kittens came from two or more litters. The seven adult deprived cats were chosen from local vendors after being identified as older animals by their large size. Two of these cats had been in the laboratory colony an additional 7 to 15 months before the onset of deprivation. All of the deprived animals were monocularly deprived for 4 weeks, and recordings were carried out immediately thereafter. Of the five normal animals, three were 39 weeks old at the time of recording and two were adults of unknown age.

Recording procedures. Animal preparation and single unit recording methods were similar to those described elsewhere (Spear et al., 1980b; Spear and Tong, 1980). Briefly, the cat was anesthetized with halothane in $50 \% \mathrm{O}_{2} / 50 \% \mathrm{~N}_{2} \mathrm{O}$ during surgical procedures. Paralysis was maintained throughout recording with intravenous gallamine triethiodide. The cat was artificially respired via a tracheal cannula, and end-expired $\mathrm{CO}_{2}$ was monitored and kept at approximately $4 \%$. Body temperature was held at $37.5^{\circ} \mathrm{C}$ with a heating pad. Heart rate was monitored continuously, and EEG was recorded through the cortical microelectrode (using appropriate low-pass amplifier filters) and checked periodically. All wound edges were infiltrated with a long-lasting local anesthetic. During recording, the animal was respired with $25 \% \mathrm{O}_{2} / 75 \%$ $\mathrm{N}_{2} \mathrm{O}$.

The deprived eye of each lid-sutured animal was opened at the beginning of the recording session and checked to be sure there were no corneal opacities. The pupils were dilated and accommodation was blocked by topical application of $1 \%$ ophthalmic atropine sulfate. The corneas were protected with contact lenses. A tangent screen was placed in front of the animal at a distance of $57 \mathrm{~cm}$ from the nodal points of the eyes. The position of the area centralis of each eye was plotted on the tangent screen (Pettigrew et al., 1979) and the eyes were focused on the screen with spectacle lenses.

Holes were made in the skull over LS and striate cortex, and the dura was cut and retracted. The skull openings were covered with an agar/sucrose gel to maintain cortical temperature and reduce pulsations. Extracellular recordings were made with varnished tungsten microelectrodes that had impedances of 50 to 70 megohms measured with a $135-\mathrm{Hz}$ sine wave input. Action potentials were amplified and led to an oscilloscope and audio monitor.

Neuronal sampling procedures. A number of precautions were taken to ensure that recording conditions and sampling procedures were as similar as possible in LS and striate cortex. First, we attempted to record from both areas of cortex in each cat, and the order of recording from the two areas was counterbalanced within each age group. This strategy was successful in all of the deprived kittens, in four of the cats deprived as adults, and in three of the normal adult cats. In the few remaining cats, recordings were made from only one cortical area.

Second, all recordings in both cortical areas were made contralateral to the deprived eye. Contralateral rather than ipsilateral recordings were chosen because the independence of effects of monocular deprivation in LS cortex and striate cortex has been established most clearly in the hemisphere contralateral to the deprived eye (Tong and Spear, 1980). In addition, since the contralateral eye dominates neurons in both LS and striate cortex of normal cats, any loss of response to the deprived eye would be most evident in the contralateral hemisphere.

Third, the traverse of the microelectrode penetrations and the single neuron sampling procedures were kept as similar as possible in the two cortical areas. To avoid disproportionate sampling of individual ocular dominance bands (Hubel and Wiesel, 1963; Shatz et al., 1977), microelectrode penetrations in both striate and LS cortex were made tangential to the cortical surface. To accomplish this in striate cortex, the microelectrode was angled $30^{\circ}$ from vertical, pointing anteriorly in the sagittal plane, and traversed the medial wall of the posterolateral gyrus. In LS cortex, the microelectrode was angled 30 to $60^{\circ}$ from vertical, pointing medially in the coronal plane, and traversed the medial wall of the middle suprasylvian sulcus. Additionally, in both cortical areas a minimum distance of $100 \mu \mathrm{m}$ separated consecutively sampled neurons.

Fourth, the same range of visual field eccentricities was sampled in both cortical areas. Only cells that had receptive field centers within $20^{\circ}$ of the area centralis and near the zero horizontal meridian were included in the sample. Such cells were readily sampled in each cortical area because of the layout of the topographic organization (Palmer et al., 1978; Tusa et al., 1978) combined with the placement of the microelectrode penetrations in each area.

Receptive field and ocular dominance measurement. Receptive field and ocular dominance properties of each isolated neuron were determined using hand-held stimuli consisting of light and dark bars, edges, and spots that were flashed or moved in various directions through the receptive field. Since our primary interest was in cortical ocular dominance, the detailed receptive field organization was not determined for each neuron. However, pains were taken to determine the stimulus that produced the optimal response of the cell for each eye independently. The optimal stimulus was then presented alternately to each eye, and repeated presentations were made while the relative response to each eye was assessed from the audio monitor.

Each cell was assigned to one of seven ocular dominance categories (Hubel and Wiesel, 1962): 1, driven exclusively by the contralateral eye; 2 , strongly dominated by the contralateral eye; 3 , slightly dominated by the contralateral eye; 4 , driven about equally by either eye; 5 , slightly dominated by the ipsilateral eye; 6 , strongly dominated by the ipsilateral eye; and 7 , driven exclusively by the ipsilateral eye. Since judgment of these categories was subjective, periodic checks were made during the course of the experiments to ensure that the criteria for classification remained the same among the three experimenters. In addition, each experimenter recorded from every cat and from both cortical areas. There were no significant differences in the results obtained by the three experimenters.

Histology. In every cat, the final electrode penetration in each cortical area was marked with two small electrolytic lesions. This allowed later reconstruction of the electrode tracks to verify that they were in the intended cortical area (see below). Marking every electrode penetration would have been more advantageous for determining the cortical layers in which cells were recorded. However, we were concerned that multiple lesions would adversely affect subsequent recordings due to damage to afferents, disruption of blood supply, or spreading depression. This was particularly a concern in LS cortex, where many penetrations sometimes were required to obtain an adequate sample of cells.

At the end of each recording session, the animal was given a lethal dose of pentobarital sodium and was perfused transcardially with $0.9 \%$ saline followed by $10 \%$ formol/saline. Each cortical area was blocked and frozen-sectioned $(52 \mu \mathrm{m})$ in the plane of its electrode tracks, mounted on slides, and stained with cresyl violet.

The location of each electrode track was related to the one marked by electrolytic lesions. In addition, when possible, the layers in which cells were recorded was determined. The layer analysis was performed primarily for electrode tracks in which electrolytic lesions were made. Tissue shrinkage was calculated from the distance between the electrolytic lesions in the section, and the depth of cells at recording was related to the lesions to determine the cortical layer in which the cell was located. Layer analysis also was performed for a few tracks without electrolytic lesions if the tracks coursed almost entirely within a single layer. In these cases, only cells from the long portion of the track that stayed within one layer were included in the analysis. Cortical layers were distinguished using the criteria of Otsuka and Hassler (1962).

\section{Results}

\section{Location of penetrations}

Data were obtained from 56 microelectode penetrations into striate cortex, of which 55 were located histologically. Some cells recorded in one of the penetrations (from a cat deprived at 4 weeks of age) were found to be in area 18 and were discarded from the sample. All other neurons in the histologically located penetrations were verified to be within area 17 . The penetration 
that was not located in histological material was assumed to be in area 17 because it was made near another penetration found to be in striate cortex in the same animal.

Data were obtained from 107 penetrations in LS cortex, of which 98 were located histologically. These penetrations were between stereotaxic coordinates $\mathrm{A}+8$ and $\mathrm{P}-4$ and traveled down the medial wall of the middle suprasylvian sulcus; therefore, they traversed the posteromedial lateral suprasylvian (PMLS) visual area of Palmer et al. (1978). Most penetrations also terminated clearly within the PMLS area in the medial half of the sulcal fundus. However, 22 penetrations terminated in the lateral half of the sulcal fundus, and 133 cells recorded in that region may have been in the posterolateral lateral suprasylvian (PLLS) visual area (Palmer et al., 1978). Nevertheless, it is likely that these cells were in the PMLS area because they were located posteriorly in the middle suprasylvian sulcus and because previous anatomical (Heath and Jones, 1971) and physiological (Spear and Baumann, 1975) studies suggest that this region also is part of the PMLS area. In any case, we found no difference in the results from these cells and the remainder of the sample; therefore, all are included in the results to be presented.

The nine penetrations not located histologically were made near penetrations found to be in the PMLS area of LS cortex. Therefore, they are assumed to be located within the PMLS area as well.

\section{Responsiveness and receptive field properties}

In striate cortex, a total of 990 cells were studied (mean of $38 / \mathrm{cat}$ ), of which $85 \%$ responded to visual stimulation. In LS cortex, a total of 869 cells were studied (mean of $35 /$ cat), of which $77 \%$ were visually responsive. For each cortical area, there were no significant differences among any of the groups of cats in either sample size or percentage of responsive cells.

Although no quantitative measures were performed, those cells that responded to the deprived eye appeared to have normal receptive fields through that eye. All common functional types normally found in both cortical areas were represented, and responses to the deprived eye often were brisk and consistent. Undoubtedly, this was so because all of the deprived animals had some period of normal visual experience between the time of eye opening and the onset of monocular deprivation. Consequently, those cells that retained responses to the deprived eye had qualitatively normal receptive fields.

\section{Ocular dominance}

Pooled ocular dominance distributions. Figure 1 shows the ocular dominance distributions for all responsive cells recorded in each group of cats. In both striate and LS cortex of normal cats, most cells were binocularly driven and there was an overall dominance by the contralateral eye.

This pattern of ocular dominance was altered dramatically by 4 weeks of monocular deprivation early in life. For both cortical areas, the greatest effects of monocular deprivation occurred in the animals deprived at 4 weeks of age. In these animals, nearly all responsive cells were driven exclusively by the nondeprived eye. At 12 and 18 weeks of age, the effects of 4 weeks of monocular deprivation were progressively less severe, although they still were clearly present in both cortical areas.

Following monocular deprivation at 26 weeks of age or older, the ocular dominance distributions in LS cortex appeared to be relatively normal. However, in area 17 , cats deprived at 26 and 35 weeks of age appeared to have a smaller percentage of binocularly driven cells than normal and there continued to be an abnormal overall dominance by the ipsilateral, nondeprived eye.

Quantitative analyses of ocular dominance. The ocular dominance results were quantified and subjected to statistical analyses by calculating several measures for each animal and comparing the measures for animals in each of the deprived groups with those for normal animals. For the statistical comparisons, Mann-Whitney tests (one-tailed) were carried out using the measure from each animal as a single observation.

Three different measures of ocular dominance were used. First, the percentage of responsive cells dominated by the ipsilateral eye (ocular dominance classes 5, 6, and 7) was calculated for each cat. This provides a measure of the extent

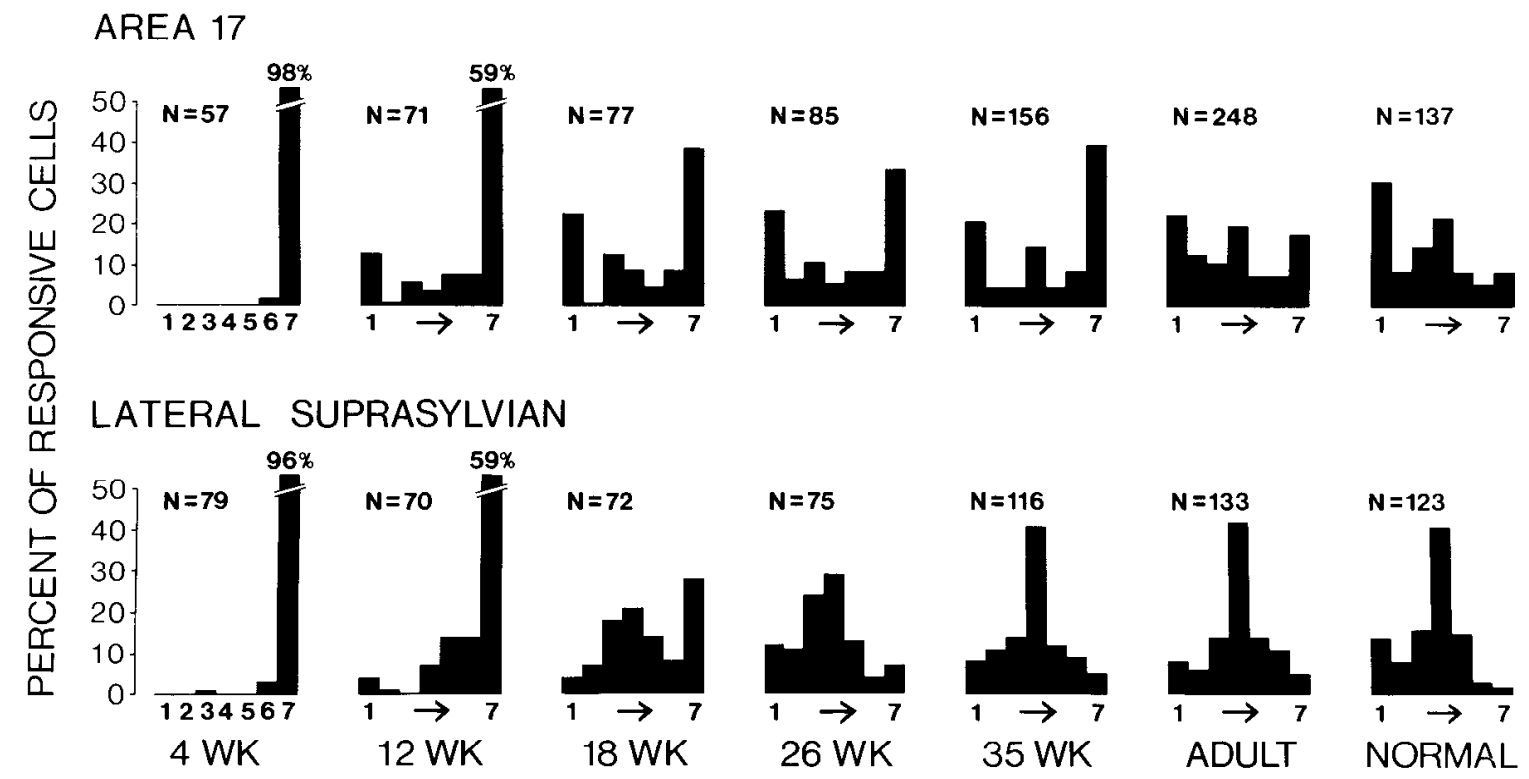

Figure 1. Ocular dominance histograms for neurons in area 17 and LS visual area of normal cats (right) and cats given 4 weeks of monocular deprivation at different ages. Ages at the bottom indicate deprivation onset. The scale beneath each histogram represents the degree of ocular dominance. Cells in ocular dominance classes 1 and 7 were driven exclusively by the contralateral (deprived) or ipsilateral (nondeprived) eye, respectively. Cells in classes 2 to 6 were binocularly driven with different degrees of dominance by each eye (see "Materials and Methods"). $N=$ total number of responsive cells sampled in each cortical area of each group of cats. 
to which the nondeprived eye of monocularly deprived cats comes to dominate cells in comparison to normal animals. Second, the percentage of responsive cells dominated by the contralateral eye (ocular dominance classes 1,2 , and 3 ) was calculated to provide a measure of the extent to which the deprived eye has lost control of cortical cells. Third, the percentage of binocularly driven cells (ocular dominance classes 2 to 6) was calculated because previous studies (Hubel and Wiesel, 1970; Olson and Freeman, 1980) have suggested that a loss of binocular cells represents an intermediate stage of effects of monocular deprivation. ${ }^{4}$

Figure 2 shows the results for these three measures in striate cortex. For all three measures, the effects of monocular deprivation decreased progressively following onsets from 4 to 18 weeks of age and then reached a plateau following onsets from 18 to 35 weeks of age. Although there was a fair amount of variability from animal to animal (e.g., in the 18-week group), it is clear that abnormalities were present in striate cortex following 4 weeks of monocular deprivation beginning as late as 35 weeks of age. Thus, the percentage of cells dominated by the ipsilateral (nondeprived) eye (Fig. 2A) is significantly higher than normal following onset of deprivation at both 26 and 35 weeks of age ( $p=0.028$ in both cases). The loss of response to the contralateral (deprived) eye (Fig. 2C) was somewhat less marked, but it too is statistically significant in animals deprived as late as 35 weeks of age $(p=0.028)$. The least reliable change was in the percentage of binocularly driven striate cortex cells, but here too there is a suggestion that abnormalities were present following monocular deprivation in 35 -week-old cats $(0.029<p<0.057)$

In cats deprived for 4 weeks as adults, none of the ocular dominance measures were significantly different from normal striate cortex (Fig. 2). Therefore, for the effects we have measured in striate cortex, there exists an end of the critical period of susceptibility to monocular deprivation that occurs sometime after 35 weeks of age.

As shown in Figure 3, results in LS cortex were quite different. As in striate cortex, the effects of monocular deprivation on LS cortex decreased progressively following onsets from 4 to 18 weeks of age. However, unlike striate cortex, there was no plateau of effects after this age. Indeed, in animals deprived at 18 weeks of age, only the percentage of cells dominated by the ipsilateral (nondeprived) eye (Fig. $3 A$ ) was significantly higher than normal $(p=0.028)$. Neither the percentage of binocular cells (Fig. $3 B$ ) nor the percentage of cells dominated by the contralateral (deprived) eye (Fig. $3 C$ ) was significantly different from normal in the 18-week group. In animals deprived at 26 and 35 weeks of age, none of the measures of ocular dominance in LS cortex were significantly different from normal. Similar results were obtained in animals deprived as adults (Fig. 3). Thus, the critical period for effects of 4 weeks of monocular deprivation in LS cortex appears to end between 18 and 26 weeks of age, and even at 18 weeks of age only minor effects are evident.

Comparison of magnitudes of the abnormalities in LS and striate cortex. These results indicate that the critical period for effects of monocular deprivation ends earlier in LS cortex than in striate cortex. It is of interest to know whether or not the course of the decline in susceptibility also differs in the two areas; that is, how do the magnitudes of the abnormalities compare following deprivation at different ages? To answer this question, it is necessary to take into account differences in ocular dominance that exist between each cortical area in

${ }^{4} \mathrm{~A}$ measure of mean ocular dominance was not used because ocular dominance classes form an ordinal and not an interval scale; therefore, their average is not a meaningful measure. normal cats (Fig. 1, NORMAL). This was done by normalizing the results for each ocular dominance measure.

The normalization procedure was as follows. First, the maximum change from normal that could occur following monocular deprivation was calculated. The maximum possible change is the difference between the most extreme score that can possibly occur (designated $E$ ) and the mean normal score $(N)$ for that measure. Second, the difference between each cat's actual score $(X)$ and the mean normal score for that measure was determined. This actual change from normal was then expressed as a percentage of the maximum possible change from normal. We will refer to this as the normalized deprivation effect $(D)$. The general formula used was: $D=[(X-N) /(E-$ $N)] \times 100$. Note that the largest possible value of $D$ is $100 \%$, a value of $0 \%$ occurs if there was no difference between the deprived animal's score and the mean normal score, and a negative value of $D$ can occur if a deprived animal's score was in the opposite direction from normal than the most extreme possible score.

As an example of the normalization procedure, consider the effects of deprivation on the ability of the nondeprived eye to dominate LS cortex cells. The mean percentage of cclls dominated by the ipsilateral eye in normal cats was $20 \%$ (Fig. $3 A$ ), and the most extreme score that could occur following monocular deprivation is that all $(100 \%)$ of the cells come to be dominated by the ipsilateral (nondeprived) eye. Thus, a change from $20 \%$ to $100 \%$ responsive to the nondeprived eye is the maximum that could occur among LS cortex cells. If a deprived cat had $60 \%$ of its LS cortex cells dominated by the ipsilateral nondeprived eye, its actual change from normal would be from $20 \%$ to $60 \%$. Therefore, the normalized deprivation effect for that cat would be $[(60-20) /(100-20)] \times 100=50 \%$. Similar calculations were carried out for each measure in each cat.

Figure 4 shows the results of this analysis by plotting the mean normalized deprivation effects for each area of cortex. Following onset of monocular deprivation between 4 and 18 weeks of age, the two cortical areas showed very similar normalized deprivation effects in terms of dominance by the nondeprived eye (Fig. $4 A$ ), binocularity (Fig. $4 B$ ), and dominance by the deprived eye (Fig. $4 C$ ). For all three measures, both cortical areas had a nearly linear monotonic decrease in effects of monocular deprivation over these ages. For LS cortex, the effects of monocular deprivation continued to decrease monotonically to approximately zero following onset at 26 weeks of age. In contrast, normalized deprivation effects in striate cortex remained relatively constant for onsets between 18 and 35 weeks of age. Throughout this age range, all three ocular dominance measures in striate cortex showed $20 \%$ to $40 \%$ of the maximum possible changes from normal. There was then a second decline following deprivation onset between 35 weeks of age and adult.

These normalized results also allow a direct comparison of the effects of monocular deprivation on the two cortical areas in individual animals. Following deprivation onset at 4,12 , and 18 weeks of age, the normalized scores were similar for striate and LS cortex of each cat. This was true for each of the ocular dominance measures. In contrast, clear differences between the two cortical areas of individual cats were seen following deprivation at 26 and 35 weeks of age. In all seven animals in these two age groups, the normalized deprivation effect was larger in striate cortex than in LS cortex for all three ocular dominance measures. The small number of animals studied in each age group precluded a meaningful statistical analysis of this result. However, because there were no significant differences in results between the 26-week and 35 -week age groups, the results for the two groups could be combined to provide a larger sample size for statistical comparison of the two cortical areas. Wilcoxon matched-pairs tests for related measures were then car- 


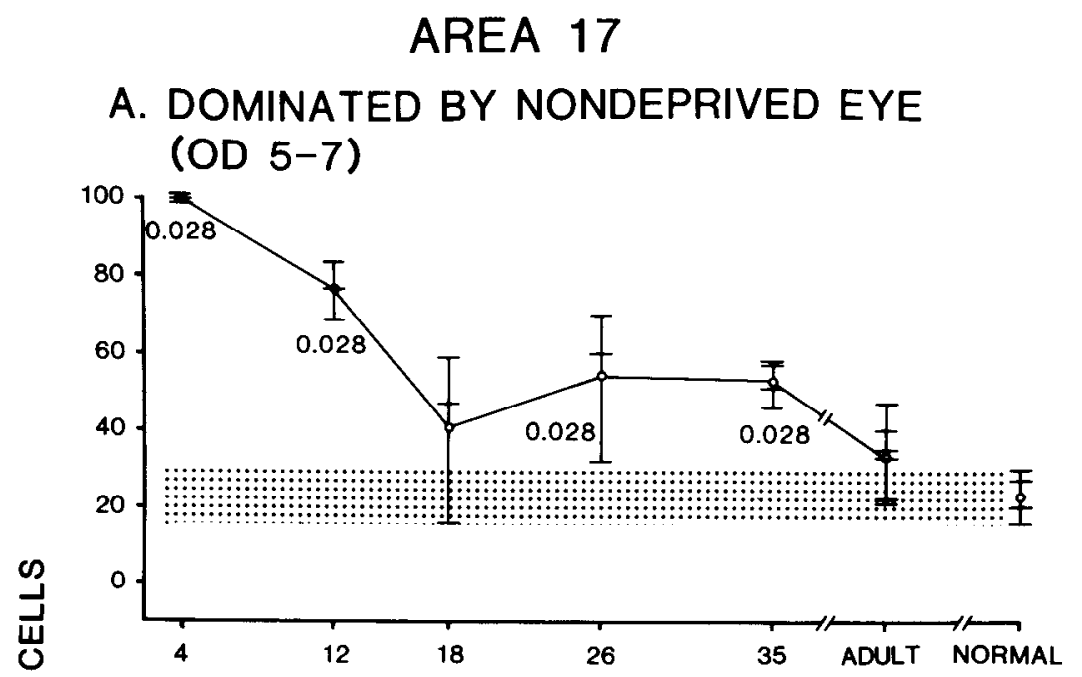

\section{B. BINOCULAR (OD 2-6)}

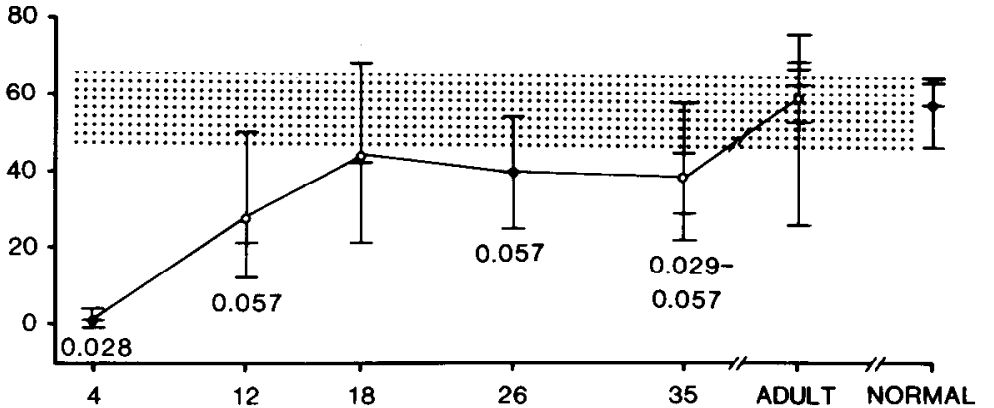

C. DOMINATED BY DEPRIVED EYE (OD 1-3)

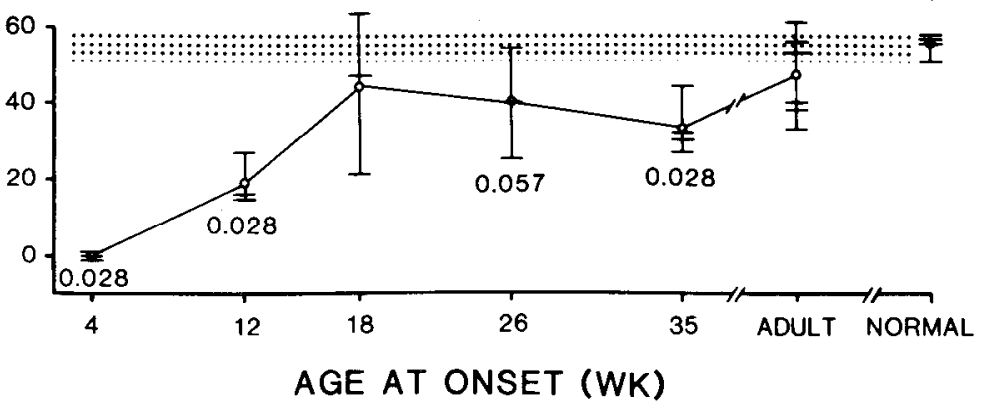

Figure 2. Summary of effects of monocular deprivation beginning at different ages on three different measures of ocular dominance in striate cortex (area 17). $A$, Percentage of responsive cells dominated by the nondeprived eye (ocular dominance classes 5-7); $B$, percentage of binocularly driven cells (classes 2 to 6 ); $C$, percentage of responsive cells dominated by the deprived eye (classes 1 to 3). Horizontal slashes represent scores for individual cats in each group and open circles represent the means of those scores. Results for normal adult cats are plotted at the far right, and the shaded area in each graph shows the total range of scores on each measure for the normal animals. Outcomes of statistical comparisons between scores for normal cats and scores for cats in each deprived group are shown by the decimals; these represent the probability that a between-group difference that large would occur by chance according to a Mann-Whitney test (one-tailed). The absence of a decimal indicates that the probability was greater than 0.1 . Note that because a rank-order statistical test has been applied to small samples, the probability cannot be less than a certain fixed value. For the sample sizes used in most of the comparisons, the minimum $p=0.028$. See the text for further description of these results.

ried out using the difference between LS and striate cortex of each cat as a single observation. These tests indicate that, for all three ocular dominance measures, the normalized deprivation effect was significantly larger in striate cortex than in LS cortex of cats deprived at 26 and 35 weeks of age $(T=0, p=$ 0.02 , two-tailed, for each measure).

\section{Cortical layers}

There is evidence that effects of monocular deprivation on cat striate cortex neurons are more severe in the upper and lower cortical layers than in layer IV (Shatz and Stryker, 1978). In addition, work in the monkey suggests that susceptibility to 


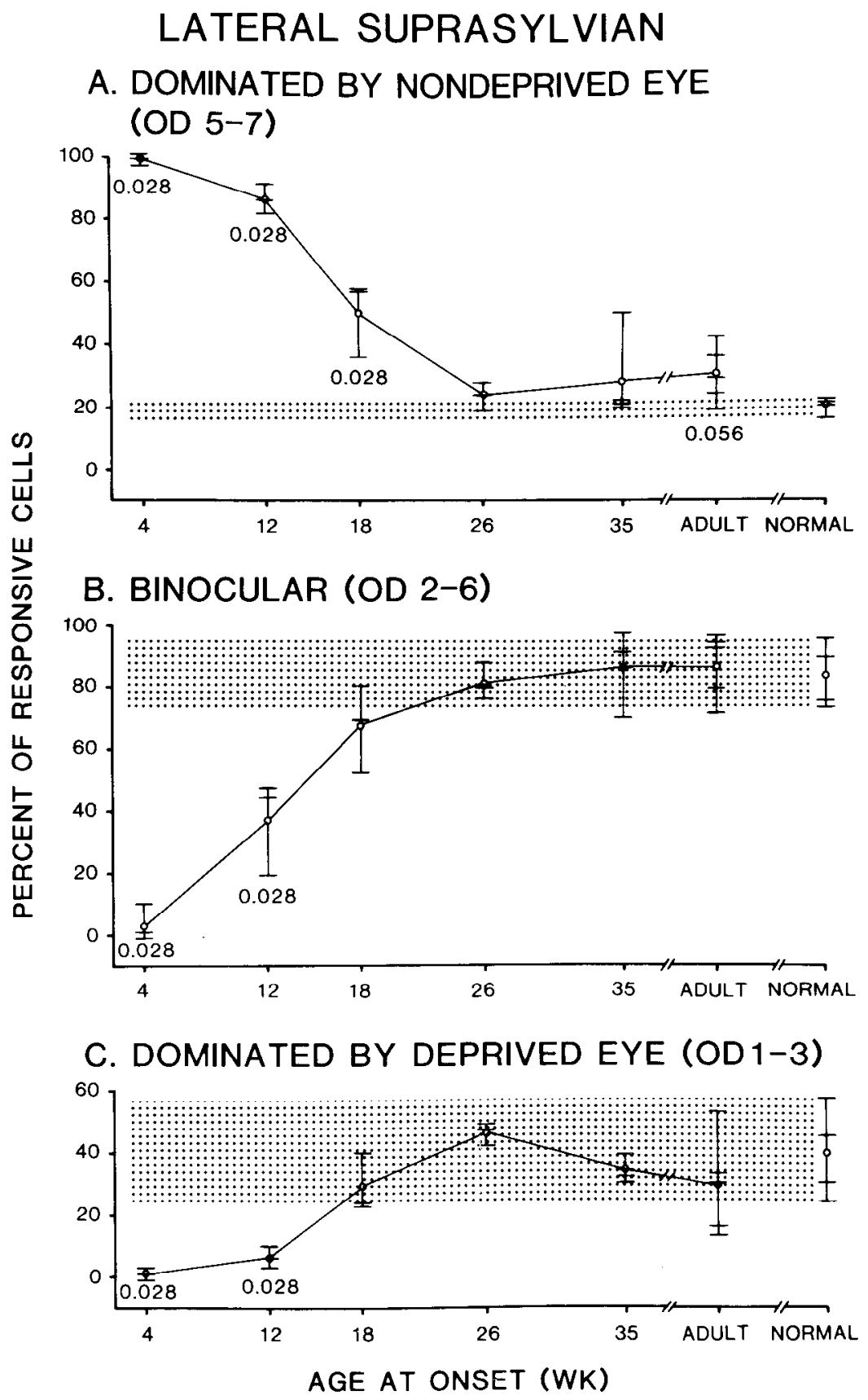

Figure 3. Summary of effects of monocular deprivation beginning at different ages on three different measures of ocular dominance in LS visual cortex. All conventions are the same as in Figure 2. See the text for further description.

monocular deprivation persists longer in the upper and lower cortical layers than in layer IV (LeVay et al., 1980). Results such as these raise the possibility that the difference between area 17 and LS cortex in susceptibility to late monocular deprivation is due to differential sampling of the various cortical layers. To address this possibility, we performed a layer analysis of ocular dominance in all cats. Only cells from penetrations in which electrode depth could be related unequivocally to layers in the histological section were used (see "Materials and Methods"). Of cells recorded in striate cortex, 40\% (394 of 990 ) could be localized as to layer; in LS cortex, only $27 \%$ (231 of 869) could definitely be placed in a particular layer.

For comparison purposes, cells were divided by layer into one of three groups: upper (layers I to III), middle (layer IV), and lower (layers $\mathrm{V}$ to $\mathrm{VI}$ ). As shown in Table I, cells were recorded in all layers in both cortical areas. However, differences in the percentage of cells sampled from the various layers clearly were evident both among age groups and between the two cortical areas. To determine whether this sampling bias affected the results, we constructed separate ocular dominance distributions for cells in layer IV and the upper and lower layers in each group of cats. For both cortical areas, this analysis revealed no difference between layer IV and non-layer IV ocular dominance distributions for any group. In addition, the differences in effects of monocular deprivation at 26 and 35 weeks of age seen in striate and LS cortex were evident in layer IV 
A. CELLS DOMINATED BY NONDEPRIVED EYE (OD 5-7)
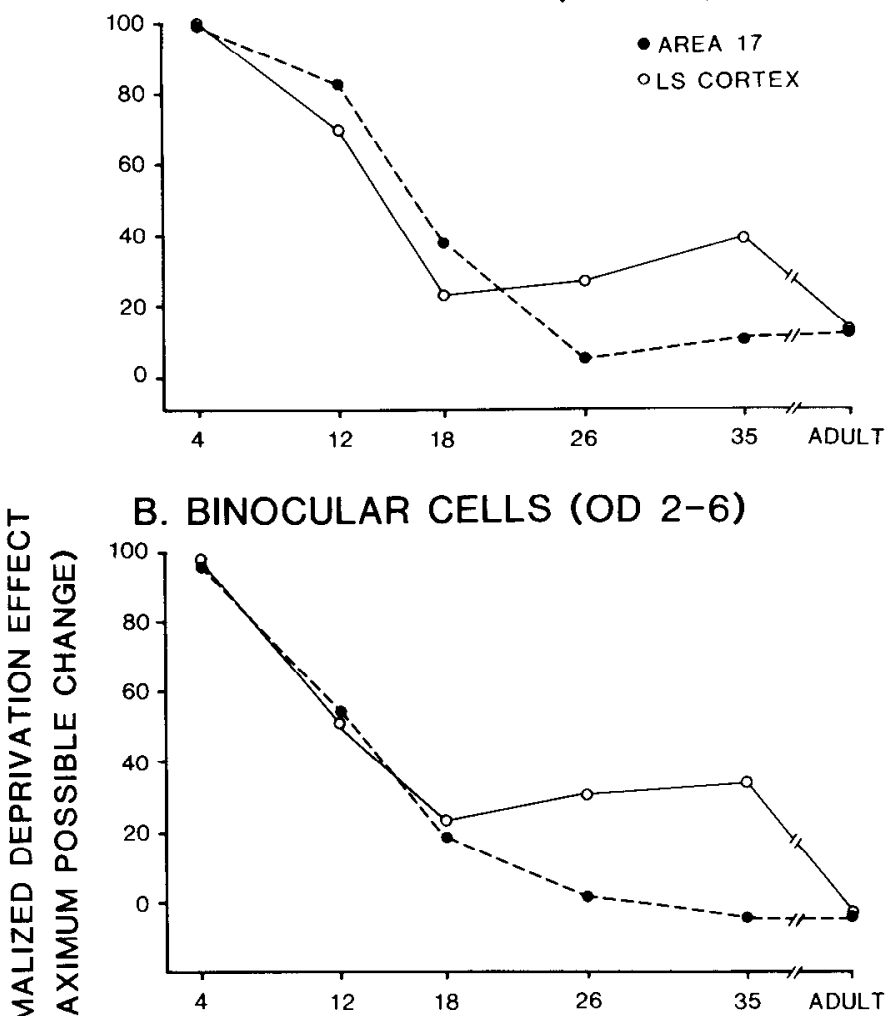

C. CELLS DOMINATED BY DEPRIVED EYE (OD 1-3)

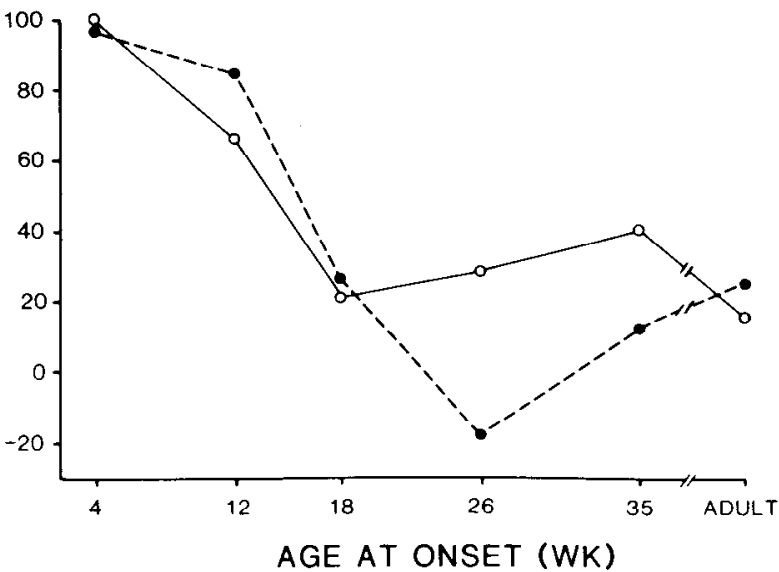

Figure 1. Direct comparison of ocular dominance changes in striate and LS visual cortex following monocular deprivation at different ages. Comparisons are made for each of three measures of ocular dominance ( $A, B$, and $C$, as in Figs. 2 and 3 ). For each area of cortex, the difference between each cat's score and the mean normal score on the measure in question was expressed as a percentage of the maximum change from normal that could occur. The formula used for this normalization procedure is described in the text. The normalized scores for cats in each group were then averaged, and these averages are shown in the figure. Thus, open circles show the mean normalized deprivation effects for cells in area 17 of cats deprived at different ages, and solid circles show the mean normalized deprivation effects for cells in LS cortex.

and in non-layer IV cells. It must be recognized that this analysis is based upon a relatively small subsample of neurons. However, it suggests that the results presented in the previous section were not due to differential sampling of layers in LS and striate cortex.
TABLE I

Percentage of located cells in each cortical layer for normal cats and cats given 4 weeks of monocular deprivation at different ages

\begin{tabular}{|c|c|c|c|c|c|c|}
\hline \multirow[b]{2}{*}{ Group } & \multicolumn{3}{|c|}{ Area 17} & \multicolumn{3}{|c|}{ Lateral Suprasylvian Area } \\
\hline & $\begin{array}{l}\text { Layers } \\
\text { I-III }\end{array}$ & Layer IV & $\begin{array}{c}\text { Layers } \\
\text { V-VI }\end{array}$ & $\begin{array}{c}\text { Layers } \\
\text { I-III }\end{array}$ & Layer IV & $\begin{array}{l}\text { Layers } \\
\text { V-VI }\end{array}$ \\
\hline 4 weeks & 10 & 0 & 90 & 49 & 20 & 31 \\
\hline 12 weeks & 0 & 57 & 43 & 68 & 32 & 0 \\
\hline 18 weeks & 13 & 11 & 76 & 29 & 46 & 25 \\
\hline 26 weeks & 25 & 31 & 44 & 14 & 81 & 6 \\
\hline 35 weeks & 26 & 34 & 40 & 23 & 57 & 20 \\
\hline Adult & 67 & 8 & 25 & 28 & 45 & 28 \\
\hline Normal & 17 & 52 & 31 & 15 & 80 & 5 \\
\hline
\end{tabular}

\section{Receptive field eccentricity}

In accord with our sampling procedures (see "Materials and Methods"), all cells in both cortical areas had receptive field centers within $20^{\circ}$ of the area centralis and near the zero horizontal meridian. However, more striate cortex cells had receptive field centers within $10^{\circ}$ of area centralis $(73 \%$ of the cells) than did LS cortex cells (37\%). This was due in part to the large size of receptive fields for LS cortex cells, which caused many cells with receptive field borders on or near the area centralis to have field centers more than $10^{\circ}$ eccentric. In any case, for both cortical areas the effects of monocular deprivation on cells with receptive field centers within $10^{\circ}$ of area centralis were similar to those on cells with field centers 10 to $20^{\circ}$ from area centralis. In addition, the differences observed between striate and LS cortex in animals deprived at 26 and 35 weeks of age were present both for cells with receptive fields near area centralis and for cells with more eccentric receptive fields.

\section{Discussion}

The main finding of this study is that the critical period for effects of monocular deprivation ends at different ages in different visual areas of cortex. Contrary to our expectation, we found that abnormalities can be induced later in striate cortex than in LS cortex. In the following two sections, we discuss the periods of susceptibility separately for IAS cortex and striate cortex and relate them to previous results in those cortical areas. In the last section, we consider the implications of the differences between the two areas of cortex.

\section{Period of susceptibility in LS cortex}

These experiments are the first to investigate the time course of the critical period for effects of visual deprivation on an area of cortex outside area 17 . The results indicate that the susceptibility of LS cortex cells to changes in ocular dominance following 4 weeks of monocular deprivation peaks at or before 4 weeks of age. Susceptibility then declines monotonically until, at 26 weeks of age, no significant effect of the deprivation is seen. Similar results are seen for all three measures of ocular dominance that were used, although the gain in dominance by the nondeprived eye appears to be the most sensitive mcasure. Indeed, results from this measure raise the possibility that some marginal effects of monocular deprivation may persist into adulthood (see Fig. 3A). However, if there are effects of monocular deprivation in LS cortex of adult cats, they are very slight and it will require large numbers of cats or longer periods of deprivation to reveal them. Under the present conditions, the critical period for effects of monocular deprivation on ocular dominance of LS cortex cells ends between 18 and 26 weeks of age.

Interestingly, these results agree well with previous studies of the period of plasticity in LS cortex following brain damage. 
Spear et al. (1980a) found that a unilateral lesion of areas 17 and 18 in adult cats produces (among other effects) a change in ocular dominance of LS cortex cells ipsilateral to the lesion, but following neonatal lesions there is a functional compensation in LS cortex and ocular dominance develops normally. Further studies showed that the critical period for this ocular dominance compensation ends between 18 and 26 weeks of age (Tong et al., 1984). Thus, the period of life during which LS cortex cells are able to alter their eye affiliation appears to be similar for both environmental (deprivation) and neural (brain damage) manipulations.

It is not clear why the alterations in ocular dominance should have such similar time courses following two such dissimilar manipulations, particularly since the nature of the inputs to LS cortex differ markedly in the two cases. In addition to the loss of inputs from ipsilateral areas 17 and 18, cats with early lesions of these areas have anomalous inputs from the lateral geniculate nucleus to LS cortex (Kalil et al., 1979; Kalil, 1984; Tong et al., 1984) that probably are not present in monocularly deprived cats (Spear and Tong, 1980; Tong and Spear, 1980). This raises the possibility that the critical period for alterations in a particular response property, such as ocular dominance, reflects some intrinsic developmental properties of LS cortex neurons.

\section{Period of susceptibility in striate cortex}

In agreement with previous studies (see Mitchell, 1981; Movshon and Van Sluyters, 1981), we found that susceptibility of striate cortex neurons to changes in ocular dominance following monocular deprivation peaks at or before 4 weeks of age. Susceptibility then declines monotonically until 18 weeks of age, after which it remains at a relatively constant level until at least 35 weeks of age. Similar results are seen with all three measures of ocular dominance that we employed although, as in LS cortex, the gain in dominance by the nondeprived eye seems to be the most sensitive measure.

Our finding that 4 weeks of monocular deprivation can cause significant abnormalities in striate cortex ocular dominance as late as 35 weeks of age was at first somewhat surprising. At the time that these experiments were initiated, it was generally accepted that the period of susceptibility to effects of monocular deprivation in area 17 was over by 12 weeks of age (Wiesel and Hubel, 1965; Hubel and Wiesel, 1970; Blakemore and Van Sluyters, 1974). However, papers that were published while our experiments were in progress concluded that the period of susceptibility ends later, either between 14 and 16 weeks (99 and 109 days) of age (Olson and Freeman, 1980) or between 26 and 30 weeks (6 and 7 months) of age (Cynader and Mitchell, 1980; Cynader et al., 1980). While these more recent studies indicate that the critical period lasts longer than previously believed, their estimates as to its end still are earlier than ours.

There are several possible reasons for these differences. Olson and Freeman (1980) used a much shorter period of deprivation (10 days) than was used in our experiments (4 weeks); thus it is perhaps not surprising that their estimate of the end of the critical period also is earlier. On the other hand, Cynader and his colleagues (Cynader and Mitchell, 1980; Cynader et al., 1980) used 3 months of monocular deprivation, and although they reported abnormalities in ocular dominance of cats deprived as late as 6 months of age, they concluded that there was no effect in 7-to 10-month-old cats. However, two observations suggest that this conclusion is unwarranted. First, since Cynader et al. (1980) do not report results from normally reared cats, specific comparisons with normal animals, which might reveal small effects of monocular deprivation, could not be made. Second, comparing the results of Cynader et al. (1980) to normal ocular dominance results reported by others does not support the conclusion that monocular deprivation is without effect in 7- to 10-month-old cats. There is general agreement in the literature that the contraluteral eye dominates the majority of neurons in normal striate cortex (e.g., Hubel and Wiesel, 1962; Blakemore and Pettigrew, 1970). Since Cynader et al. (1980) made their recordings from the hemisphere contralateral to the deprived eye, they should have observed an overall dominance by the deprived eye if there was no effect of monocular deprivation. Contrary to this expectation, the combined results from two cats deprived at 7 months of age show a flat ocular dominance distribution with a very slight dominance by the ipsilateral nondeprived eye (Fig. $2 D$ of Cynader et al., 1980). In addition, results from cats deprived at 10 months of age show an overall dominance by the ipsilateral nondeprived eye (Fig. 3 of Cynader and Mitchell, 1980). Only in a single cat deprived at 8 months of age is there an overall dominance by the contralateral deprived eye (Fig. $2 E$ of $\mathrm{Cy}$ nader et al., 1980; Fig. 3 of Cynader and Mitchell, 1980). Thus, when all of the animals studied by Cynader and his colleagues are considered, the results actually provide evidence that monocular deprivation can affect striate cortex ocular dominance in 7 - to 10 -month-old cats. This, together with the consistent effects that we observed in our 35-week-old animals (Figs. 2 and 4), indicates that the period of susceptibility of striate cortex to monocular deprivation clearly extends beyond 8 months of age.

When does the critical period end in striate cortex? Our results indicate that 4 weeks of monocular deprivation in adult animals produces no significant effects on striate cortex ocular dominance. Nevertheless, small changes in the percentage of cells dominated by the deprived and nondeprived eyes were present in these animals (see Fig. 2, $A$ and $C$ ), and it remains possible that more prolonged monocular deprivation can produce abnormalities in adult cats. Data pertinent to this question are sparse in the literature. Results from two cats suggest that 3 or 16 months of monocular deprivation in otherwise normally reared adults has no effect on ocular dominance of striate cortex cells (Wiesel and Hubel, 1963; Hubel and Wiesel, 1970). On the other hand, Singer et al. (1982) have reported that rotating one eye and suturing the other eye closed for a period of 6 months in adult cats results in abnormal responsiveness and ocular dominance among striate cortex cells. Thus, whether prolonged deprivation in adult cats can produce changes in striate cortex ocular dominance is somewhat uncertain.

\section{Comparison between striate and LS cortex}

A major objective of this study was to determine whether the time course of susceptibility to the same regime of monocular deprivation is the same in striate and LS cortex. The results indicate that both areas are most susceptible at or before 4 weeks of age and that both areas show a very similar decline in sensitivity over the next 12 weeks. However, after 18 weeks of age there is a clear divergence in the susceptibilities of the two cortical areas to the same deprivation conditions. Betweengroup comparisons with normal cats indicate that striate cortex continues to be susceptible at least until 35 weeks of age, whereas LS cortex no longer shows effects of the deprivation after 18 weeks of age. In addition, within-animal comparisons of the two cortical areas show that in every cat deprived at 26 or 35 weeks of age, the effects on all three measures of ocular dominance were greater in striate cortex than in LS cortex.

Possible artifacts. It is important to consider possible artifacts that could produce the differences between striate and LS cortex. Using the same cats for recordings in both cortical areas rules out the possibility that differences in genetic or environmental conditions could have affected the results. In addition, since the order of recording from the two cortical areas was counterbalanced across cats in each group, it is unlikely that differences in the time interval between opening the sutured 
eye and recording, or differences in the condition of the preparation, could have affected the results.

Another possibility is that if susceptibility to monocular deprivation varies among cortical layers in a given area of cortex, disproportionately sampling different layers could produce spurious differences between the two areas of cortex or even between different groups of cats for the same cortical area. There were, in fact, some differences between groups of cats and between the two cortical areas in the percentages of cells recorded from each cortical layer. However, we found no clear differences between the layers in effects of the deprivation conditions employed in these experiments. ${ }^{5}$ Moreover, the greater susceptibility of striate cortex compared to LS cortex in 26- and 35-week-old cats was observed even when results from particular layers were analyzed separately. Therefore, differential sampling of cortical layers does not seem to have produced the differences between groups or between cortical areas. Similarly, the results cannot be accounted for on the basis of differences in receptive field eccentricity for cells sampled in striate and LS cortex.

Some implications of the differences. The observation that area 17 and LS cortex have different critical periods for effects of monocular deprivation indicates that these effects occur at least partly independently in the two areas. This supports the conclusion, reached from other experiments, that abnormalities in LS cortex following visual deprivation are not due simply to abnormal inputs from areas 17, 18 or 19 (Spear and Tong, 1980; Tong and Spear, 1980; Spear et al., 1983). Rather, the thalamocortical pathways for each area appear to be affected independently. However, it is not known whether the thalamic inputs to LS cortex are affected differently from the thalamic inputs to striate cortex, or whether the thalamic inputs to the two areas are affected in the same way but the two cortical areas respond to them differently. The answer to this question will provide important information concerning the sites underlying effects of visual deprivation.

Whatever the neural sites of the differences between striate and LS cortex, these results make it increasingly clear that the mechanisms controlling developmental sequences and critical periods of plasticity in the brain are very complex. Previous studies have shown that the same region of the brain (and possibly the same neurons) can have different critical periods for effects on different response properties (Daw et al., 1978; Tong et al., 1984), and the present results show that closely related regions of cortex can have different critical periods for effects on the same response property. This multiplicity of critical periods implies a multiplicity of neural events, and possibly even of underlying mechanisms, that lead to plasticity in brain (see Tong et al., 1984, for further discussion of this issue).

A final point concerns the behavioral implications of these results. There have been many studies of the behavioral effects of monocular deprivation and attempts have been made to relate the behavioral abnormalities to abnormalities in the geniculostriate pathways. But other areas of the brain clearly suffer abnormalities following visual deprivation and, as we have shown, they can have different critical periods for these

\footnotetext{
${ }^{5}$ Shatz and Stryker (1978) did find that layer IV cells were somewhat less affected than cells in the upper and lower layers of striate cortex in monocularly deprived cats. However, they used very long deprivation beginning at the time of normal eye opening, which results in almost no upper or lower layer cells that respond to the deprived eye. The small difference in effects between layer IV and the other layers (Fig. 3 of Shatz and Stryker, 1978) may be reduced or eliminated under deprivation conditions that leave many cells responsive to the deprived eye, as was the case in all but the 4-week-old deprived group in the present experiments.
}

abnormalities. Therefore, changes in behavioral effects following onset of deprivation at different ages may be due to changes in effects on different areas of the brain rather than to changes within a single area.

\section{References}

Blakemore, C., and J. D. Pettigrew (1970) Eye dominance in the visual cortex. Nature 225: 426-429.

Blakemore, C., and R. C. Van Sluyters (1974) Reversal of the physiological effects of monocular deprivation in kittens: Further evidence for a sensitive period. J. Physiol. (Lond.) 237: 195-216.

Cynader, M., and D. E. Mitchell (1980) Prolonged sensitivity to monocular deprivation in dark-reared cats. J. Neurophysiol. 43: 1026-1040.

Cynader, M., B. N. Timney, and D. E. Mitchell (1980) Period of susceptibility of kitten visual cortex to the effects of monocular deprivation extends beyond six months of age. Brain Res. 191: 545550 .

Daw, N. W., N. E. J. Berman, and M. Ariel (1978) Interaction of critical periods in the visual cortex of kittens. Science 199: 565-567.

Heath, C. J., and E. G. Jones (1971) The anatomical organization of the suprasylvian gyrus of the cat. Ergeb. Anat. Entwicklungsgesch. 45: 1-64.

Hubel, D. H., and T. N. Wiesel (1962) Receptive fields, binocular interaction and functional architecture in the cat's visual cortex. $\mathrm{J}$. Physiol. (Lond.) 160: 106-154.

Hubel, D. H., and T. N. Wiesel (1963) Shape and arrangement of columns in cat's striate cortex. J. Physiol. (Lond.) 165: 559-568.

Hubel, D. H., and T. N. Wiesel (1970) The period of susceptibility to the physiological effects of unilateral eye closure in kittens. J. Physiol. (Lond.) 206: 419-436.

Kalil, R. E. (1984) Removal of visual cortex in the cat: Effects on the morphological development of the retino-geniculo-cortical pathway. In Development of Visual Pathways in Mammals, J. Stone, ed., pp. 257-274, Alan R. Liss, New York.

Kalil, R. E., L. Tong, and P. D. Spear (1979) Reorganization of the geniculo-cortical pathway in the cat following neonatal damage to visual cortex. Invest. Ophthalmol. Vis. Sci. Suppl. 18: 157.

LeVay, S., T. N. Wiesel, and D. H. Hubel (1980) The development of ocular dominance columns in normal and visually deprived monkeys. J. Comp. Neurol. 191: 1-52.

Mitchell, D. E. (1981) Sensitive periods in visual development. In Development of Perception: Psychobiological Perspectives, R. N. Aslin, J. R. Alberts, and M. R. Petersen, eds., Vol. 2., pp 3-44, Academic Press, Inc., New York.

Movshon, J. A., and R. C. Van Sluyters (1981) Visual neural development Annu. Rev. Psychol. 32: 477-522.

Olson, C. R., and R. D. Freeman (1980) Profile of the sensitive period for monocular deprivation in kittens. Exp. Brain Res. 39: 17-22.

Otsuka, R., and R. Hassler (1962) Über Aufbau und Gliederung der corticalen Schsphäre bei der Katze. Arch. Psychiatr. Z. Gesamte Neurol. 203: 212-234.

Palmer, L. A., A. C. Rosenquist, and R. J. Tusa (1978) The retinotopic organization of lateral suprasylvian visual areas in the cat. J. Comp. Neurol. 177: 237-256.

Pettigrew, J. D., M. L. Cooper, and G. G. Blasdel (1979) Improved use of tapetal reflection for eye position monitoring. Invest. Ophthalmol. Vis. Sci. 18: 490-495.

Shatz, C. J., and M. P. Stryker (1978) Ocular dominance in layer IV of the cat's visual cortex and the effects of monocular deprivation. J. Physiol. (Lond.) 281: 267-284

Shatz, C. J., S. Lindström, and T. N. Wiesel (1977) The distribution of afferents representing the right and left eyes in the cat's visual cortex. Brain Res. 131: 103-116.

Singer, W., F. Tretter, and U. Yinon (1982) Evidence for long-term functional plasticity in the visual cortex of adult cats. J. Physiol. (Lond.) 324: 239-248.

Spear, P. D., and T. P. Baumann (1975) Receptive-field characteristics of single neurons in lateral suprasylvian visual area of the cat. J. Neurophysiol. 38: 1403-1420.

Spear, P. D., and L. Tong (1980) Effects of monocular deprivation on neurons in cat's lateral suprasylvian visual area. I. Comparison of binocular and monocular segments. J. Neurophysiol. 44: 568-584. 
Spear, P. D., R. E. Kalil, and L. Tong (1980a) Functional compensation in lateral suprasylvian visual area following neonatal visual cortex removal in cats. J. Neurophysiol. 43: 851-869.

Spear, P. D., A. Langsetmo, and D. C. Smith (1980b) Age-related changes in effects of monocular deprivation on cat striate cortex neurons. J. Neurophysiol. 43: 559-580.

Spear, P. D., L. Tong, and C. Sawyer (1983) Effects of binocular deprivation on responses of cells in cat's lateral suprasylvian visual cortex. J. Neurophysiol. 49: 366-382.

Tong, I.., and P. D. Spear (1980) Fffects of monocular deprivation on neurons in cat's lateral suprasylvian visual area. II. Role of visual cortex and thalamus in the abnormalities. J. Neurophysiol. 44: 585604 .
Tong, L., R. E. Kalil, and P. D. Spear (1984) Critical periods for functional and anatomical compensation in the lateral suprasylvian visual area following removal of visual cortex in cats. J. Neurophysiol., in press.

'I'usa, K. J., L. A. Palmer, and A. C. Rosenquist (1978) The retinotopic organization of area 17 (striate cortex) in the cat. J. Comp. Neurol. 177: $213-236$.

Wiesel, T. N., and D. H. Hubel (1963) Single-cell responses in striate cortex of kittens deprived of vision in one eye. J. Neurophysiol. 26: $1003-1017$.

Wiesel, T. N., and D. H. Hubel (1965) Comparison of the effects of unilateral and bilateral eye closure on cortical unit responses in kittens. J. Neurophysiol. 28: 1029-1040. 